\title{
INEQUALITIES FOR THE GAMMA FUNCTION RELATING TO ASYMPTOTIC EXPANSIONS
}

\author{
G. Allasia, C. GiORdAnO AND J. PeČARIĆ
}

\begin{abstract}
Many inequalities for the gamma function can be deduced from monotonicity or convexity properties of $\log \Gamma(x)$ and related functions involving finite sums of the Stirling asymptotic series. Considering a particular case of a generalization of this classical expansion, we deduce further convexity results and inequalities which are similar to some other ones related to the usual form of the Stirling series. We give, among other things, inequalities which overvalue $\log \Gamma(x)$, whereas the corresponding finite sums of the classical expansion undervalue it or vice versa. Moreover we obtain bilateral inequalities also for the digamma and the polygamma functions. Finally, a few extensions of Gautschi-type inequalities are discussed.
\end{abstract}

Mathematics subject classification (2000): 33B15, 26D07, 26A51.

Key words and phrases: gamma function, inequalities, convex functions, asymptotic expansions, Euler-Mclaurin formulas.

\section{REFERENCES}

[1] G. Allasia, C. Giordano And PeČARIĆ, Hadamard-type inequalities for (2r)-convex functions with applications, Acc. Sc. Torino, Atti Sc. Fis. 133 (1999), 1-14.

[2] H. Alzer, On some inequalities for the gamma and psi functions, Math. Comp. 66 (1997), 373-385.

[3] J. Bustoz, M. E. M. Ismail, On Gamma Function Inequalities, Math. Comp. 47 (1986), 659-667.

[4] F. Casolaro, C. D' Aniello and M. P. Giovine, Asymptotic Inequalities for $\lg \Gamma(x)$, Atti Sem. Mat. Fis. Univ. Modena XLIV (1996), 303-312.

[5] N. Elezović, C. GioRdano And J. PeČARIĆ, The best bounds in Gautschi's inequality, Math. Ineq. Appl. 3, 2 (2000), 239-252.

[6] W. GAUTSCHI, Some elementary inequalities relating to the gamma and incomplete gamma function, J. Math. Phys. 38 (1959), 77-81.

[7] W. GAUTSCHI, The incomplete gamma functions since Tricomi, Atti Conv. Lincei Roma, "Tricomi's Ideas and Contemporary Applied Mathematics", Accademia Nazionale dei Lincei, Roma, 147 (1998), 203-237.

[8] D. KeRSHAw, Some extensions of W. Gautschi's inequalities for the gamma function, Math. Comp. 41, 164 (1983), 607-611.

[9] M. MerkLE, Conditions for convexity of a derivative and some applications to the gamma function, Aequationes Math. 55 (1998), 273-280.

[10] M. MERKLE, Logarithmic convexity and inequalities for the gamma function, J. Math. Anal. Appl. 203 (1996), 369-380.

[11] M. MERKLE, Convexity, Schur-convexity and bounds for the gamma function involving the digamma function, Rocky Mountain J. of Math. 28 (1998), 1053-1066.

[12] M. E. MuldoOn, Some monotonicity properties and characterizations of the gamma function, Aequationes Math. 18 (1978), 54-63.

[13] F. W. J. Olver, Asymptotics and Special Functions, Academic Press, New York, 1974.

[14] J. PeČArIĆ, G. Allasia AND C. GIORDANo, Convexity and the Gamma Function, Indian J. Math. 41, 1 (1999), 79-93.

[15] J. F. StEFFEnSEN, Interpolation, Chelsea, New York, 1950, pp. 119-138. 